\title{
State-of-the-Art Series on Precision Medicine in Respiratory Diseases
}

\author{
Christoph Lange ${ }^{a-d}$ Salman Siddiqui ${ }^{e}, f$ \\ ${ }^{a}$ Research Center Borstel, Clinical Infectious Diseases, German Center for Infection Research (DZIF), Borstel, and \\ ${ }^{\mathrm{b}}$ International Health/Infectious Diseases, University of Lübeck, Lübeck, Germany; ' Department of Medicine, \\ Karolinska Institute, Stockholm, Sweden; d Department of Medicine, University of Namibia, School of Medicine, \\ Windhoek, Namibia; ${ }^{e}$ Department of Infection, Immunity and Inflammation, University of Leicester, and \\ ${ }^{\mathrm{f}} \mathrm{NIHR}$ Respiratory Biomedical Research Unit, Glenfield Hospital, Leicester, UK
}

\section{Key Words}

Precision medicine $\cdot$ Personalized medicine

Respiratory diseases are a leading cause of morbidity and mortality worldwide. Despite the enormous costs associated with the establishment of a correct diagnosis and medical treatment, disease modification and cure is not possible for many patients with chronic respiratory diseases or malignancies by currently available therapies. Diagnosis of chronic respiratory diseases and lung cancer is often delayed, treatment often consists of suboptimal therapies, and in many settings patients do not receive adequate treatments at all because of unavailability or a poor disease prognosis. Additionally, some patients experience adverse events from available treatments or side effects that limit their utility.

In recent years, major advances in the understanding of the pathogenesis of respiratory diseases have been made that allow tailoring medical treatment to the characteristics of each individual patient. Unraveling the genetic basis of diseases and molecular pathways that lead to the disease manifestation and the influence of environmental factors on disease expression has opened the possibility to develop prevention strategies and improve the diagnosis and treatment of affected patients on a personalized level. Precision medicine [1], in a broad sense defined as 'prevention and treatment strategies that take individual variability into account' [2], aims to classify individuals into subpopulations with different susceptibilities to a particular disease, with different disease pathogeneses and manifestations, as well as different clinical courses and responses to specific therapies. The aim of precision medicine is to improve individual patient care by linking the advances in medical research and computer science, which is expressed in a US Presidential Initiative that allocated 215,000,000 USD in the 2016 fiscal year for a Precision Medicine Initiative [3].

Stratification of patients into groups with different molecular bases and phenotypic disease expressions is not a novel concept in medicine. It has been developed over many years by classification of diseases into types and subtypes, which are usually reclassified when advances in the knowledge about the origin, manifestation and prognosis of a disease provide better insight.

Novel technologies, such as automated whole-genome sequencing of human and microbial DNA and mRNA (which can now be performed at the level of single cells), transcriptome gene expression profiles, high-throughput proteomics, metabolomics, lipidomics and imaging approaches with quantitative regional functional biomarkers are currently revolutionizing the classification of phenotypic disease subtypes. Improvements in the under-

\section{KARGER}

E-Mail karger@karger.com

www.karger.com/res (c) 2016 S. Karger AG, Basel

0025-7931/16/0924-0197\$39.50/0
Christoph Lange, MD

Clinical Infectious Diseases, Research Center Borstel Parkallee 35

DE-23845 Borstel (Germany)

E-Mail clange@fz-borstel.de 
standing of regulatory networks and key metabolic pathways enable modulation of complex immune responses and the design of immune checkpoint inhibitory therapies, which have already entered the stage of clinical applications. Therapeutic drug monitoring will potentially allow providing individual dosage-tailored therapies to optimize treatment effects and to minimize adverse events, and biomarkers are being identified, evaluated and applied in clinical practice to monitor treatment responses and to define the duration of therapy in individual patients. However, the 'penalty' of scientific advance in precision medicine has been the explosion of complex data, and a unique implementation challenge of precision medicine is to have federated knowledge management systems with harmonized bioinformatics and data visualization techniques.

There is no doubt that in time, precision medicine will lead to diagnostic applications to identify those who will mostly benefit from specific therapies, while saving expenses and avoiding effects of treatments for those who will not.

Respiration is focusing on four disease areas with a state-of-the-art series. Sharma et al. [in preparation] report on the implementation of precision medicine for the care of lung cancer, where individualization of management is most advanced in respiratory medicine. They review important findings for the understanding of the molecular mechanisms that lead to the genesis and progression of lung cancer and the development of targeted therapies and immune checkpoint inhibitor therapy that have dramatically improved the prognosis for patients with specific subtypes of this malignancy. In this issue of Respiration, Salzer et al. [4] are providing a comprehensive outlook on the implementation of precision medicine for the care of patients with chronic respiratory infectious diseases, e.g. tuberculosis, nontuberculous mycobacterial pulmonary diseases and chronic pulmonary aspergillosis. They compare current to expert management standards and provide an outlook for future appli- cations of innovative diagnostics and novel therapeutic applications, including host-directed therapies that will lead to a substantially improved prognosis for patients to be cured from these diseases. Heaney et al. [in preparation] challenge the traditional concept of stepwise treatment of the airway diseases (asthma and chronic obstructive pulmonary disease) endorsed by international guidelines. Novel developments in the field of airway inflammometry, biomarkers and companion diagnostics characterizing endotypes of severe asthma and chronic obstructive pulmonary disease that stratify the response to biologics and small-molecule inhibitors, behavioral psychology and the advent of quantitative tools to characterize adherence to inhalers using digital technologies as well as the important impact of comorbidities on airway disease expression are critically evaluated. Richeldi et al. [in preparation] provide an overview of precision medicine in idiopathic pulmonary fibrosis, novel molecular endotypes that underpin fibrogenesis and repair, results of some pivotal phase 3 studies of idiopathic pulmonary fibrosis and the movement towards classifying fibrotic lung disease based upon molecular endotypes in a cutting-edge review of this fast-moving clinical field.

Although not all patients can be cured from respiratory diseases, the implication of precision medicine will continue to improve the prognosis of our patients. Precision medicine implies that personal care for the individual patient leads to a better life. However, a patient-centered holistic concept that goes beyond the scientific advances of precision medicine and keeps humanity in the focus of our work will be a truly personal one.

\section{Acknowledgements}

C.L. is supported by the German Center for Infection Research (DZIF). This editorial presents independent research funded by the National Institute for Health Research (NIHR). The views expressed are those of the authors and not necessarily those of the NHS, the NIHR or the Department of Health.
References
1 Jameson JL, Longo DL: Precision medicine personalized, problematic, and promising. $\mathrm{N}$ Engl J Med 2015;372:2229-2234.

- Collins FS, Varmus H: A new initiative on precision medicine. N Engl J Med 2015;372: 793-795.

3 The White House, 2015; https://http://www. whitehouse.gov/precision-medicine.
-4 Salzer HJF, Wassilew N, Köhler N, Olaru ID, Günther G, Herzmann C, Kalsdorf B, Sanchez-Carballo P, Terhalle E, Rolling T, Lange C, Heyckendorf J: Personalized medicine for chronic respiratory infectious diseases: tuberculosis, nontuberculous mycobacterial pulmonary diseases, and chronic pulmonary aspergillosis. Respiration 2016;92:199-214.

\section{(1)}

$+2$

\footnotetext{
Respiration 2016;92:197-198 DOI: $10.1159 / 000450625$
}

Lange/Siddiqui 